\title{
Thymoma-associated Progressive Encephalomyelitis with Rigidity and Myoclonus (PERM) with Myasthenia Gravis
}

\author{
Satoshi Morise ${ }^{1}$, Masataka Nakamura ${ }^{1}$, Jun-ichi Morita ${ }^{2}$, Kousuke Miyake ${ }^{1}$, \\ Takenobu Kunieda ${ }^{1}$, Satoshi Kaneko ${ }^{1}$ and Hirofumi Kusaka ${ }^{1}$
}

\begin{abstract}
We report a case of a 72-year-old woman who initially presented with symptoms of bulbar myasthenia and was positive for anti-acetylcholine receptor antibodies. She subsequently developed painful muscle spasms, myoclonus, and stiffness. Thymoma was detected, and both anti-glycine receptor and anti-glutamic acid decarboxylase antibodies were found. She was diagnosed with thymoma-associated progressive encephalomyelitis with rigidity and myoclonus (PERM). She experienced marked improvement after thymectomy followed by plasma exchange and intravenous immunoglobulin and prednisolone. This case suggests that thymectomy followed by sufficient immunosuppression may be useful in the treatment of thymoma-associated PERM. Myasthenia gravis may develop in thymoma-associated PERM patients.
\end{abstract}

Key words: progressive encephalomyelitis with rigidity and myoclonus, stiff-person syndrome, glycine receptor, glutamic acid decarboxylase, myasthenia gravis

(Intern Med 56: 1733-1737, 2017)

(DOI: 10.2169/internalmedicine.56.7979)

\section{Introduction}

Progressive encephalomyelitis with rigidity and myoclonus (PERM), part of the spectrum of stiff-person syndrome (SPS), is a rare neurological disorder characterized by axial and limb rigidity, painful muscle spasms, hyperekplexia, brainstem signs, and autonomic dysfunction (1). Anti-glycine receptor (GlyR) antibodies are typically found in PERM patients with or without a tumor, although some patients also have anti-glutamic acid decarboxylase (GAD) antibodies (2). We herein report a case of thymomaassociated PERM positive for GlyR, GAD, and acetylcholine receptor $(\mathrm{AChR})$ antibodies that was successfully treated with thymectomy followed by immunosuppressive therapy.

\section{Case Report}

A 72-year-old woman visited a local hospital with a 1month history of dysarthria and chewing difficulties with masticatory fatigue in 2015. The attending neurologist strongly suspected that she had myasthenia gravis (MG) and thus prescribed pyridostigmine bromide $60 \mathrm{mg}$ twice a day; however, she could not keep taking the medicine because of side effects such as nausea and vomiting. One week later, she experienced difficulty walking because of myoclonic jerks of her left leg. Occasionally, extensor spasms of the trunk (opisthotonus-like position) also occurred. Twelve days later, she was admitted to her local hospital. Anti-AChR antibodies were positive, and dysarthria significantly improved after intravenous administration of edrophonium chloride. Saline was also injected intravenously before edrophonium chloride to exclude the placebo effect, but it did not improve her symptoms at all. She was diagnosed with MG, and treatment with tacrolimus ( $3 \mathrm{mg}$ /day) was started. However, she began to experience painful muscle spasms and stiffness of the lower extremities as well as myoclonus of the upper and lower extremities, and finally she became to be bedridden. Clonazepam and diazepam were then introduced, and some limited improvement in the painful spasms and myoclonus occurred, but these symptoms gradually worsened again. Therefore, she was transferred to our hospital for further investigation and treatment approximately two months after

${ }^{1}$ Department of Neurology, Kansai Medical University, Japan and ${ }^{2}$ Department of Neurology, Komatsu Hospital, Japan Received for publication July 14, 2016; Accepted for publication October 30, 2016 Correspondence to Dr. Masataka Nakamura, nakamuma@hirakata.kmu.ac.jp 
the onset of symptoms.

On admission, she was alert and well oriented without dementia (mini mental state examination [MMSE] was 27/30). A neurological examination revealed abnormalities in ocular movements, such as slow saccade and vertical gaze restriction, ptosis after sustained upgaze for 20 seconds, dysarthria, and dysphagia. Marked rigidity of her neck and spasticity of the lower limbs were also noticed. It was impossible to move her knee joints passively. Myoclonus and painful muscle spasms spreading to all muscles of the upper and lower extremities were easily elicited by touching or noise. Hyperreflexia in both feet was observed, and pathological reflexes were positive in both legs. There were no sensory disturbances.

A laboratory examination showed elevated C-reactive protein $(3.52 \mathrm{mg} / \mathrm{L})$. The thyroid function was normal, but the level of anti-thyroid peroxidase and anti-thyroglobulin antibodies were elevated. The anti-GAD antibody level was $74,000 \mathrm{U} / \mathrm{mL}$ in serum and $610 \mathrm{U} / \mathrm{mL}$ in the cerebrospinal fluid (CSF). Anti-AChR antibodies were positive at a titer of $0.5 \mathrm{nmol} / \mathrm{L}$. Serum antibodies to $\mathrm{Yo}, \mathrm{Hu}, \mathrm{Ri}$, amphiphysin, $\mathrm{Ma} 2 / \mathrm{Ta}$, voltage-gated potassium channel (VGKC), and $\mathrm{N}-$ methyl-D-aspartate (NMDA) receptors were all negative. A CSF analysis indicated normal values.

Surface electromyography (EMG) revealed continuous contraction of both agonist and antagonist muscles in the extremities during myoclonic jerks. The results of a nerve conduction study and needle EMG were normal. There was no decrease in the compound muscle action potential on repetitive stimulation of the median nerve at a frequency of 3 Hz. Tibial somatosensory evoked potentials (SEPs) did not show giant SEPs. Electroencephalogram showed diffuse low-amplitude background activity without epileptic discharge. Chest computed tomography (CT) identified a large mass with punctate calcification in the anterior mediastinum (Figure A). The cranial and spinal cord magnetic resonance imaging (MRI) findings were normal, except for acute thoracolumbar vertebral compression fracture due to muscle spasms.

As the clinical history and investigations didn't exclude tetanus, penicillin $\mathrm{G}(1,200,000 \mathrm{U} /$ day for 10 days) and intravenous immunoglobulin (IVIG) therapy $(2.5 \mathrm{~g} /$ day for 3 days) were attempted but did not improve her symptoms. Based on a tentative diagnosis of paraneoplastic SPS associated with a thymoma, IVIG therapy $(0.4 \mathrm{~g} / \mathrm{kg} /$ day for 5 days) was initiated. The serum anti-GAD antibody level decreased to $44,000 \mathrm{U} / \mathrm{mL}$, but her muscle stiffness and painful spasms worsened. Therefore, we performed extended thymectomy three weeks after admission. A histological examination showed the thymoma to consist of abundant epithelial cells with irregular nuclei and mature lymphocytic cells, so the tumor was diagnosed as a type B2 tumor according to the World Health Organization (WHO) classification and Masaoka stage II.

After the operation, the myoclonus and painful muscle spasms were extremely severe, and her MMSE score de- creased to 10/30. Her GAD antibody titers remained high, both in the serum $(51,000 \mathrm{U} / \mathrm{mL})$ and CSF $(600 \mathrm{U} / \mathrm{mL})$. In addition, anti-GlyR antibodies were revealed to be positive in both the serum and CSF, corroborating PERM. We therefore treated her with plasma exchange (PLEX). Her painful muscle spasms dramatically started to improve, and she was able to maintain a sitting position following completion of the seventh PLEX cycle. However, the lower limb spasticity persisted, and one month later, she progressively developed left horizontal gaze palsy and psychiatric symptoms (delusions or sudden onset of crying spells). Treatment with another 7 PLEX cycles followed by IVIG therapy $(0.4 \mathrm{~g} / \mathrm{kg} /$ day for 5 days) resulted in further improvements in the limb spasticity and myoclonus. As a result, she was able to start walking with a walker. Five months after the illness onset, she relapsed with painful spasms and was treated with intravenous methylprednisolone pulse therapy $(1,000 \mathrm{mg} /$ day for 3 days) followed by maintenance therapy with oral prednisolone (PSL, $50 \mathrm{mg} / \mathrm{day}$ ). Consequently, the painful spasms and myoclonus disappeared, and the PSL dosage was tapered. Subsequently, her left horizontal gaze palsy and psychiatric symptoms improved, and her MMSE improved to 22/30. Two months later, she was able to walk with a walking stick. Her GAD antibody titers showed significant improvement, and the anti-AChR antibodies became normalized following immunomodulatory therapy (Figure B).

\section{Discussion}

SPS was initially described in 1956 as a disorder characterized by progressive rigidity and painful muscle spasms leading to gait difficulties (3). Subsequently, SPS was established as an autoimmune disorder, most frequently associated with antibodies against GAD (4). PERM is similar to SPS with rigidity, stimulus-sensitive spasms, myoclonus, hyperekplexia, and autonomic disturbance but with additional brainstem or other neurological defects (5). The GlyR, which is prominent in the spinal cord and brainstem, is recognized as a possible target for autoantibodies in patients with PERM (6). The present patient showed progressive muscle stiffness, painful muscle spasms, myoclonus, brainstem defects, and long-tract signs. These clinical symptoms and positive results for the anti-GlyR and anti-GAD antibodies were consistent with PERM.

There have been a few reports of the association of thymoma with PERM $(7,8)$. These previous cases (Patients 1 and 2) are summarized in Table, along with the present case (Patient 3). These three patients comprised one man and two women. Anti-GlyR antibodies were detected in Patients 2 and 3. Patients 1 and 3 showed antibodies against GAD and AChR. Patient 3 had also thyroid antibodies. Thymectomy was performed in all three cases, and these histologically defined thymomas were suggested to be type B1 or B2 tumors according to the WHO histologic classification criteria. In these three cases, the effects of immunosuppressive therapy (corticosteroids, IVIG, PLEX) introduced before 
A

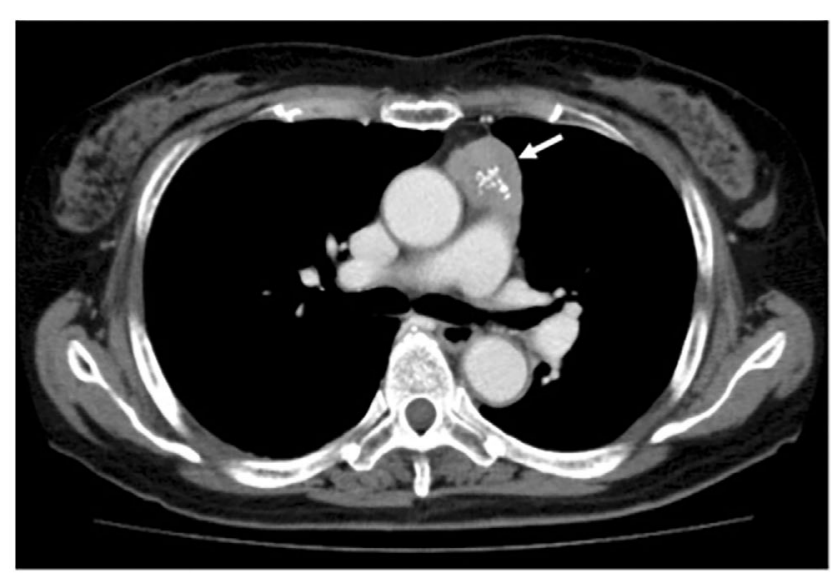

B

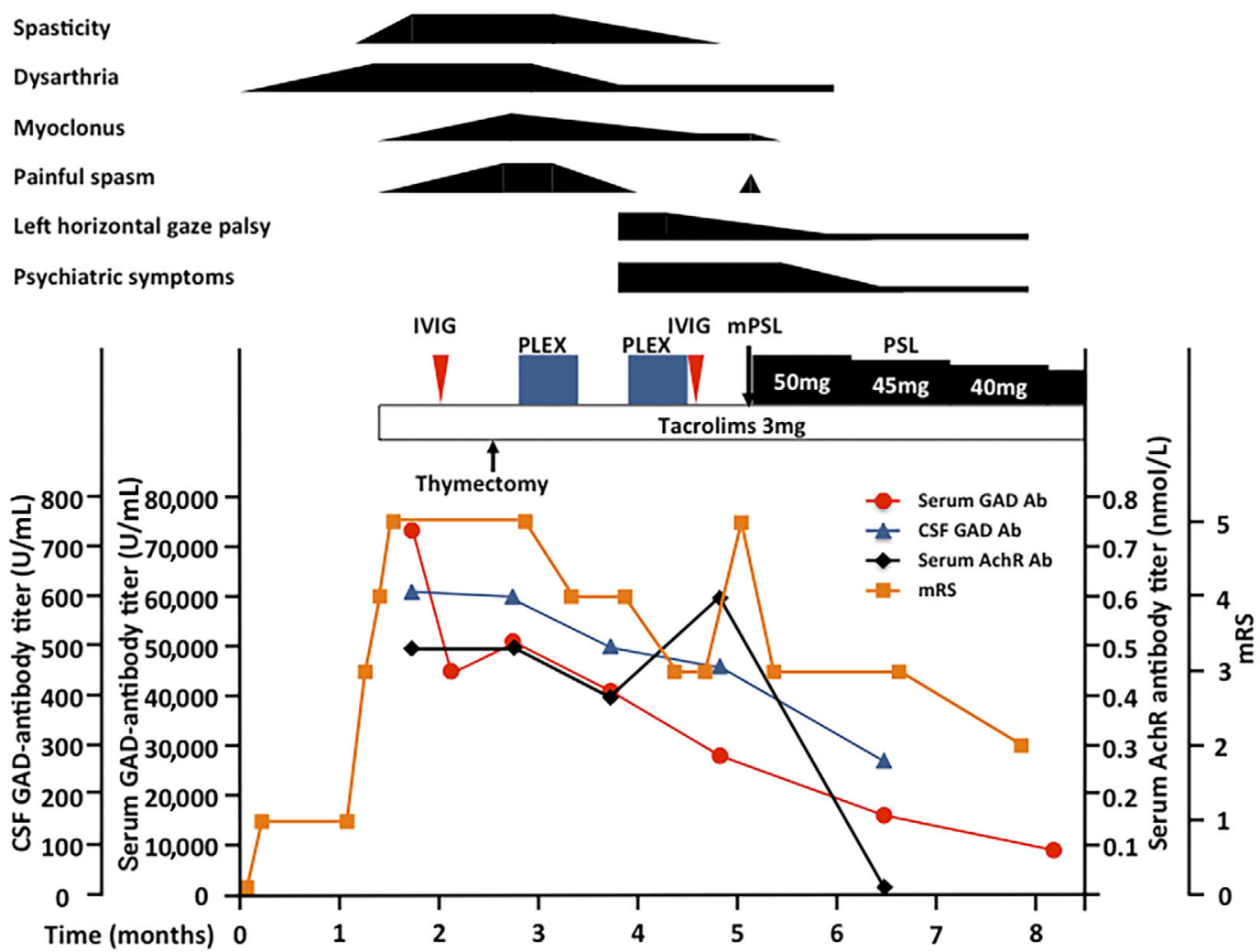

Figure. A: A thoracic computed tomography scan showing an anterior mediastinal mass with punctate calcification (arrow). B: The correlation between the disease activity and GAD and AChR antibodies titers. Neither IVIG nor thymectomy improved her clinical symptoms. The serum GAD antibody titer was decreased by IVIG but later increased again. After the initiation of PLEX, spasticity, dysarthria, myoclonus, and the painful spasms began to improve, but new symptoms, such as left horizontal gaze palsy and psychiatric symptoms, appeared. After another round of PLEX followed by IVIG, the spasticity and painful spasms disappeared, but the painful spasms recurred later. However, these painful spasms, psychiatric symptoms, horizontal gaze palsy, myoclonus, and dysarthria gradually resolved with mPSL pulse and oral PSL therapy. The serum and CSF GAD antibody titer decreased after thymectomy followed by PLEX, IVIG, mPSL pulse, and oral PSL. The serum AChR antibody titer normalized. GAD: glutamic acid decarboxylase, AChR: acetylcholine receptor, CSF: cerebrospinal fluid, IVG: intravenous immunoglobulin, PLEX: plasma exchange, mPSL: methylprednisolone, PSL: prednisolone, mRS: modified Rankin Scale 
Table. Summary of Clinical Features, Laboratory Findings, and Outcome.

\begin{tabular}{|c|c|c|c|}
\hline & Patient 1 & Patient 2 & Patient 3 \\
\hline \multicolumn{4}{|l|}{ Demographics } \\
\hline Age at onset (y) & 52 & 49 & 72 \\
\hline Sex & Female & Male & Female \\
\hline \multicolumn{4}{|l|}{ Symptoms } \\
\hline Initial symptoms & Awkardness (left leg) & Pain (right leg) & Dysarthria, Chewing difficulties \\
\hline Myoclonus & + & + & + \\
\hline Stiffness & + & + & + \\
\hline Spasm & + & + & + \\
\hline Hyperreflexia & + & - & + \\
\hline Ocular movements & - & - & $\begin{array}{l}\text { Slow saccade, Vertical gaze restriction, } \\
\text { Horizontal gaze palsy }\end{array}$ \\
\hline Brain stem sign & & Dysarthria, Diplopia & Dysarthria, Dysphagia \\
\hline Neuropsychiatric symptoms & + & + & + \\
\hline Dysautonomia & - & + & - \\
\hline Myasthenia & - & - & + \\
\hline \multicolumn{4}{|l|}{ Antibodies } \\
\hline GAD (serum/CSF) & $+/+$ & $-\mu$ & $+/+$ \\
\hline GlyR (serum/CSF) & na/na & $+/ n a$ & $+/+$ \\
\hline Amphiphysin & - & - & - \\
\hline AchR & + & na & + \\
\hline Other Abs & - & - & TPO-Abs, Tg-Abs \\
\hline \multicolumn{4}{|l|}{ Pathology } \\
\hline WHO classification & type B1 & type B1 & type B2 \\
\hline \multicolumn{4}{|l|}{ Therapy/response } \\
\hline \multicolumn{4}{|l|}{ Before thymectomy } \\
\hline IVIG & - & - & Poor \\
\hline PLEX or DFPP & Poor & Poor & - \\
\hline Corticosteroids & Poor & - & - \\
\hline Thymectomy & Good & Excellent & Poor \\
\hline \multicolumn{4}{|l|}{ After thymectomy } \\
\hline IVIG & Excerent & - & Fair \\
\hline PLEX & - & - & Excerent \\
\hline Corticosteroids & - & Good & Good \\
\hline Relapse & - & - & + \\
\hline Outcome (mRS) & $5 \rightarrow 0$ & $5 \rightarrow 0$ & $5 \rightarrow 2$ \\
\hline Reference & (8) & (7) & Present case \\
\hline
\end{tabular}

GAD: glutamic acid decarboxylase, GlyR: AchR: glycine receptor, acetylcholine receptor, Abs: antibodies, CSF: cerebrospinal fluid, IVIG: intravenous immunoglobulin, PLEX: plasma exchange, DFPP: double-filtration plasmapheresis, mRS: modified Rankin Scale, na: not available

thymectomy were not sufficient. After thymectomy, Patients 1 and 2 showed a good response to IVIG and corticosteroids, respectively. Our case responded well to PLEX but relapsed. IVIG did not seem to have any marked effect. Although which immunotherapeutic regimen is the most effective against PERM remains to be established, sufficient immunosuppressive therapy should be considered after thymectomy in cases with thymoma-associated PERM.

Initially, our case had symptoms of bulbar myasthenia and was diagnosed with MG based on the serum anti-AChR antibody level and the results of the edrophonium test, but no diurnal variation was observed. Patient 1 was also positive for anti-AChR antibodies but didn't have MG-like symptoms. Carvajal-González et al. reported 2 patients with MGlike weakness among 33 GlyR-positive PERM patients, in- cluding 1 with a history of Hodgkin's lymphoma and 1 with a thymoma. Neither had AChR or Muscle-Specific Kinase (MuSK) antibodies (5), suggesting that anti-GlyR antibodies themselves may cause symptoms similar to MG. The development of myasthenic symptoms among some cases of SPS associated with a thymoma has also been reported (9-11). For these reasons, it is crucial to rule out MG in thymomaassociated PERM patients presenting with eye movement abnormalities or bulbar symptoms.

Although we were unable to evaluate the anti-GlyR antibody titers repeatedly, the levels of both anti-AChR and anti-GAD antibodies paralleled the patient's clinical progress, with marked reductions in the levels following successful treatment. Therefore, in the present case, these thymoma-associated autoantibodies may have been associ- 
ated with her symptoms.

In conclusion, we presented a case of successful therapy for PERM with combination treatment consisting of thymoma removal and sufficient immunosuppressive therapy followed by maintenance with PSL, which successfully protected the patient against relapse. MG may develop in PERM patients associated with a thymoma.

The authors state that they have no Conflict of Interest (COI).

\section{Acknowledgement}

We thank Dr. Josep Dalmau (Department of Neurology, University of Barcelona), Dr. Keiko Tanaka (Department of Neurology, Kanazawa Medical University), and Dr. Osamu Watanabe (Department of Neurology and Geriatrics, Kagoshima University Graduate School of Medical and Dental Sciences) for measuring the Anti-GlyR, anti-NMDA receptor, and anti-VGKC antibodies, respectively.

\section{References}

1. Meinck HM, Thompson PD. Stiff man syndrome and related conditions. Mov Disord 17: 853-866, 2002.

2. Baizabal-Carvallo JF, Jankovic J. Stiff-person syndrome: insights into a complex autoimmune disorder. J Neurol Neurosurg Psychiatry 86: 840-848, 2015.

3. Moersch FP, Woltman HW. Progressive fluctuating muscular rigidity and spasm ("stiff-man" syndrome); report of a case and some observations in 13 other cases. Proc Staff Meet Mayo Clin 31: 421-427, 1956.

4. Baizabal-Carvallo JF, Jankovic J. Movement disorders in autoimmune diseases. Mov Disord 27: 935-946, 2012.

5. Carvajal-González A, Leite MI, Waters $P$, et al. Glycine receptor antibodies in PERM and related syndromes: characteristics, clinical features and outcomes. Brain 137: 2178-2192, 2014.

6. Mas N, Saiz A, Leite MI, et al. Antiglycine-receptor encephalomyelitis with rigidity. J Neurol Neurosurg Psychiatry 82: 13991401, 2011.

7. Clerinx K, Breban T, Schrooten M, et al. Progressive encephalomyelitis with rigidity and myoclonus: resolution after thymectomy. Neurology 76: 303-304, 2011.

8. Uehara T, Murai H, Yamasaki R, et al. Thymoma-associated progressive encephalomyelitis with rigidity and myoclonus successfully treated with thymectomy and intravenous immunoglobulin. Eur Neurol 66: 328-330, 2011.

9. Nicholas AP, Chatterjee A, Arnold MM, Claussen GC, Zorn GL Jr, Oh SJ. Stiff-persons' syndrome associated with thymoma and subsequent myasthenia gravis. Muscle Nerve 20: 493-498, 1997.

10. Tanaka H, Matsumura A, Okumura M, Kitaguchi M, Yamamoto S, Iuchi K. Stiff man syndrome with thymoma. Ann Thorac Surg 80: 739-741, 2005.

11. Thomas S, Critchley P, Lawden M, et al. Stiff person syndrome with eye movement abnormality, myasthenia gravis, and thymoma. J Neurol Neurosurg Psychiatry 76: 141-142, 2005.

The Internal Medicine is an Open Access article distributed under the Creative Commons Attribution-NonCommercial-NoDerivatives 4.0 International License. To view the details of this license, please visit (https://creativecommons.org/licenses/ by-nc-nd/4.0/).

(C) 2017 The Japanese Society of Internal Medicine http://www.naika.or.jp/imonline/index.html 